\title{
Untersuchung über den Normalbereich des Gesamteiweißes und der Eiweißfraktionen des Liquor cerebrospinalis nach elektrophoretischer Trennung auf Celluloseacetatfolie
}

\author{
Von J. Mertin, H. Wisser und P. Doerr \\ Aus dem Max-Planck-Institut für Psycbiatrie, Mïnchen
}

(Eingegangen am 16. Februar 1971)

Herrn Professor Dr. G. Peters zum 65. Geburtstag gewidmet

Zur Gewinnung von Normalwerten werden an einer Stichprobe von 60 Frauen und 80 Männern im Alter zwischen 16 und 74 Jahren
im Liquor cerebrospinalis das Gesamteiweiß sowie die verschiedenen Eiweißfraktionen nach elektrophoretischer Trennung ermittelt. Die
benutzten Auswahlkriterien für das Normalkollektiv werden diskutiert. Das Gesamteiweiß wird nach Fällung mit Trichloressigsäure
mit der Biuret-Methode bestimmt. Die Konzentrierung des Liquors für die Elektrophorese wird mit Überdruckfiltration durchgeführt.
Anschließend erfolgt die Auftrennung des Konzentrates durch Mikrozonenelektrophorese auf Celluloseacetattolie. Nach Anfärbung mit
Amidoschwarz 10 B werden die Fraktionen photometrisch bestimmt. Die Reproduzierbarkeit der angewandten Methode wird angegeben.

Studies on the normal concentration range of total protein and the total protein fractions of the cerebrospinal fluid by electrophoresis on cellulose acetate

In order to obtain a range of normal values, random samples of cerebrospinal fluid from 60 women and 80 men between the ages of 16 and 74 years, were analysed for total protein, and different protein fractions were determined by electrophoresis. The criteria for the selection of the normal collective are discussed. Total protein was measured by the biuret method after precipitation with trichloracetic acid. The fluid was concentrated by ultrafiltration, then resolved by microzonal electrophoresis on cellulose acetate membranes. After staining with amido black $10 \mathrm{~B}$, the fractions were measured photometrically. The reproducibility of the method is reported.

Die Verwendung von Celluloseacetatfolien anstatt Papier als Trägermaterial bei der elektrophoretischen Trennung führt zu einer Vergrößerung der Albuminund Verkleinerung der $\gamma$-Globulinfraktion (1). Dies ist bedingt durch geringere Adsorption des Eiweißes auf der Celluloseacetatfolie und die dadurch verminderte Albuminschleppe (2). Normalwerte aus Untersuchungen mit Papier als Träger können somit als Beurteilungsgrundlage bei dieser Technik nicht herangezogen werden. Dies war der Anlaß für die Gewinnung von Normalwerten mit der verränderten Arbeitsweise.

Eine Schwierigkeit bei dieser Untersuchung ist das Ziehen einer repräsentativen Stichprobe von gesunden Probanden bzw. von Probanden ohne pathologische Liquorveränderungen. Eine Probengewinnung bei einer kleinen Anzahl von gesunden freiwilligen Versuchspersonen, wie sie von anderer Seite durchgeführt wurde (3), hat den Nachteil der kleinen Zahl. Fragen wie Alters- und Geschlechtsabhängigkeit der analysierten Bestandteile sind so nicht zu klären. Andererseits bringt das von uns eingeschlagene Vorgehen eine gewisse Unsicherheit im Entscheidungsbereich normal pathologisch.

\section{Methodik}

Die Gesamteiweißbestimmung wurde mit der Biuret-Methode nach Fällung der Proteine mit 20proz. Trichloressigsäure durchgeführt. Infolge der niedrigen Eiweißkonzentration des Liquors ist zur elektrophoretischen Auftrennung des Eiweißes eine Konzentrierung erforderlich. Dazi wurde das Verfahren der Uberdruckfiltration benutzt (4). Die Elektrophorese wurde mit der Boskamp-Kammer ${ }^{1}$ ) auf Celluloseacetatfolie durchgeführt.
Gesamteiweißbestimmung

\section{Reagenzien}

Wenn nicht anders angegeben, wurden Reagenzien der Firma Merck vom Reinheitsgrad p. a. verwendet.

1. Physiolog. Natriumchloridlösung

2. 20proz. Trichloressigsäure

3. Biuret-Reagenz

In etwa $400 \mathrm{ml} 0,2 \mathrm{~N} \mathrm{NaOH}$ werden $9,0 \mathrm{~g}$ Kaliumnatriumtartrat gelöst und anschließend $3 \mathrm{~g}$ gepulvertes Kupfersulfat vollständig in einem $1000 \mathrm{ml}$ Meßkolben zur Lösung gebracht. Dann werden $0,5 \mathrm{~g}$ Kaliumjodid hinzugefïgt. Die Lösung wird bis zur Marke mit $0,2 \mathrm{~N} \mathrm{NaOH}$ aufgefüllt.

4. Standard

Stammlösung: Kontrollprobe mit bekanntem Eiweißgehalt (z. B. Labtrol'2)

Arbeitslösung: Es werden eine 1:50 und eine 1:100 Verdünnung der Kontrollprobe angesetzt, indem $200 \mu \mathrm{l}$ bzw. $100 \mu \mathrm{l}$ des Kontrollserums mit physiolog. $\mathrm{NaCl}-\mathrm{Lösung}$ auf $10 \mathrm{ml}$ verdünnt werden.

Geräte

1. Mikrolitersystem Eppendorf ${ }^{3}$ )

2. Photometer Eppendorf mit $2 \mathrm{~cm}$ Mikroküvetten ${ }^{3}$ )

3. Filter $546 \mathrm{~nm}^{3}$ )

\section{Arbeitsweise}

In die Reaktionsgefäßc ${ }^{3}$ ) werden $500 \mu 1$ Probelösung bzw. $500 \mu \mathrm{l}$ der Arbeitslösungen des Standards pipettiert und anschließend mit $250 \mu \mathrm{l}$ 20proz. Trichloressigsäure versetzt. Die Proben

1) Boskamp Gerätebaiu K.-G., Hersel/Bonn.

2) Dade Reagents Inc., Miami, Fla., USA.

3) Eppendorf Gerätcbau, Nethcler und Hinz GmbH, HamburgHummelsbüttel. 
werden dann 3 Min. am Rüttler geschüttelt, 10 Min. stehen gelassen und anschließend 1 Min. zentrifugiert. Der Uberstand wird abgegossen und verworfen. Flüssigkeitsreste werden mit einem spitzen Tupfer entfernt. Zum Niederschlag werden $100 \mu 1$ physiolog. Natriumchloridlösung pipettiert und dieser mit einem Rührstab ${ }^{3}$ ) möglichst homogen verteilt. Anschließend werden $500 \mu \mathrm{l}$ Biuret-Reagenz zugegeben. Das Reaktionsgemisch wird 5 Min. am Rüttler durchmischt und nach 30 minütigem Stehen die Extinktion bei $546 \mathrm{~nm}$ gegen den Reagenzienleerwert gemessen.

Der Reagenzienleerwert wird mit $100 \mu l$ 0,9proz. NaCl-Lösung und $500 \mu l$ Biuret-Reagenz angesetzt.

Licgt die Gesamteiveißkonzentration über $2 \mathrm{~g} / 1$, so werden die Proben mit physiolog. Natriumchloridlösung verdünnt und die Verdünnung zur Analyse angesetzt. Bei jeder Serie werden zwei 1:100 Verdünnungen einer Serumkontrollprobe mit konstantem, aber unbekanntem Eirveißgehalt zur statistischen Qualitätskontrolle mitgeführt.

Berecbnumg

$$
c_{P}=\frac{c_{S t}}{E_{S t}} E_{P}
$$

$\mathrm{c}=$ Konzentration

$\mathrm{E}=$ Extinktion

$P=$ Probe

St $=$ Standard

\section{Elektrophorese}

\section{Reagenzien und Zubebör}

1. Ultrafilter Typ SM 12136 aus Cellulosenitrat mit einem Durchmesser von $20 \mathrm{~mm}^{4}$ ). (Vor Gebrauch werden die Filter, die in 25-30proz. Alkohol aufbewahrt werden, $30 \mathrm{Min}$. in demineral. Wasser gelegt.)

2. Celluloseacetatfolien $25,5 \times 14,5 \mathrm{~mm}^{1}$ )

3. Puffer-, Färbe-, Entfärbe- und Transparenzlösung werden als Fertiglösungen der Firma Boskamp benutzt. Nach Angaben der Herstellerfirma haben sie folgende Zusammensetzungen ${ }^{5}$ ):

Pufferlösung: Michaelispuffer $\mathrm{pH} 8,6$

$$
\begin{aligned}
& \text { Veronalnatrium } 58,86 \mathrm{~g} \\
& \text { Natriumacetat } 38,86 \mathrm{~g} \\
& 0,1 \mathrm{~N} \mathrm{HCl} 360 \mathrm{ml} \\
& \text { dest. Wasser ad } 61
\end{aligned}
$$

Als Arbeitslösung wird eine Verdünnung von drei Teilen Puffer mit zwei Teilen demineralisiertem Wasser benutzt.

Färbelösung: gesättigte Lösung von Amidoschwarz $10 \mathrm{~B}$ in Methanol-Eisessig $9 / 1(\nabla / v)$

Entfärbelösung: Methanol-Eisessig $9 / 1(\nabla / v)$

Transparenzlösung: Dioxan-Isobutanol $7 / 3(\mathrm{v} / \mathrm{v})$

\section{Geräte}

1. Filtrationsgerät MD-20 mit Metallschlauch ${ }^{4}$ )

2. Stickstoffbombe mit Reduzierventil

3. Mikrophor-Elektrophoresekammer ${ }^{1}$ )

4. Streifenträger für Folien $25,5 \times 14,5 \mathrm{~mm}$ (Farbkennzeichen Schwarz)

5. Auftragstempel

6. Vokam-Netzgerät ${ }^{6}$ )

7. Extinktionsschreiber 3 mit Integralschreiber ${ }^{7}$ )

4) Sartorius Membranfilter GmbH, Göttingen. (Das Filtrationsgerät MD-20 mit einem Aufgußraum von $3 \mathrm{ml}$ ist nach Auskunft der Herstellerfirma seit 2 Jahren nicht mehr Bestandteil des Vetkaufsprogramms. Für den gleichen $Z_{\text {weck }}$ wurde von seiten der Firma das Filtrationsgerät SM 16223 mit einem Aufgußraum von $20 \mathrm{ml}$ und einem Filterdurchmesser von $25 \mathrm{~mm}$ empfohlen.)

5) Bedienungsanleitung: Celluloseacetat-Elektrophorese der Firma Boskamp

6) Shandon Labortechnik GmbH, Frankfurt/Main

7) Carl Zeiss, Oberkochen/Württ.

\section{Arbeitsweise}

Konzentrierung der Probe:

Je nach der Höhe des bestimmten Gesamteiweißgehalts werden 0,5 bis $1,0 \mathrm{ml}$ Liquor in das Filtrationsgerät MD-20 pipettiert. Die Filtration wird mit nachgereinigtem Stickstoff von 14 atii durchgeführt. 'Diese Art der Konzentrierung dauert bei sachgemäßer Durchführung durchschnittlich $30 \mathrm{Min}$.

\section{Elektrophoretische Trennung:}

Nach Beendigung der Druckfiltration wird der Rückstand in 10 bis $20 \mu \mathrm{l}$ der oben angegebenen Verdünnung des Michaelispuffers aufgenommen. Aufnehmen der Probe und Auftragen auf die Folie werden mit dem Auftragstempel durchgeführt. Aus Sicherheitsgründen wird die gleiche Probe auf einer Folie dreifach aufgetragen. Vorbehandlung, Färben, Entfärben, Aufziehen der Folien auf Objektträger und Behandlung im Transparenzbad werden ohne Änderungen nach den Angaben der Firma Boskamp ${ }^{5}$ ) durchgeführt. Die Pherogramme werden bei einer konstanten Spannung von $250 \mathrm{~V}$ und einer Trennzeit von $25 \mathrm{Min}$. erstellt. Die Trennstrecke beträgt 36 bis $38 \mathrm{~mm}$. Die Auswertung erfolgt photometrisch mit dem Extinktionsschreiber 3 mit Integtalschreiber?).

\section{Probengewinnung}

Die Liquorentnahme erfolgte bei allen Patienten durch Lumbalpunktion. Diese wurde in den meisten Fällen im Sitzen durchgeführt; ein kleinerer Teil der Patienten, bei denen die Punktion im Rahmen einer Myelographie erfolgte, wurde auf dem horizontal stehenden Röntgentisch in Bauchlage punktiert.

Nach komplikationsloser Punktion wurden in allen Fällen die für die Zellzahlbestimmung und die Eiweißuntersuchungen benötigten Liquormengen getrennt aufgefangen: die ersten Tropfen, etwa $0,5 \mathrm{ml}$, zur Zellzahlbestimmung, die nächsten 2-2,5 ml zur Bestimmung des Gesamteiweißes, für die Liquorelektrophorese, die Pandy- und die Normomastixreaktion.

Kurz vor der Lumbalpunktion wurden jeweils einige $\mathrm{ml}$ venöses Blut für die Serumelektrophorese abgenommen.

\section{Normalwertbestimmung}

\section{Kriterien zur Auswabl der Patienten für die Normalwertbestimmung}

Die zur Normalwettermittlung herangezogenen Liquorbefunde wurden aus dem Kollektiv aller seit 1967 in unserer Klinik vorgenommenen Liquoruntersuchungen ausgewählt. Berücksichtigt wurden ausschließlich stationär aufgenommene Patienten der neurologischen und psychiatrischen Abteilung, die meist zur Diagnostik - einschließlich neuroradiologischer und neurophysiologischer Untersuchungen - überwiesen worden waren. Aus dem Gesamtkollektiv wurden ausgeschlossen:

1. Patienten, die aufgrund ihrer Anamnese und des klinischen Befundes Anhaltspunkte für eine bekanntermaßen mit Liquorveränderungen einhergehende Erkrankung zeigten (5-8),

2. Patienten mit einem entzündlichen Blutbild und/oder einer Dysproteinämie,

3. Patienten mit einem pathologischen Liquorbefund: Zellzahl größer als 12/3 Zellen im lumbalen Liquor, Pandyreaktion positiv, pathologische Normomastixkurve.

Liquorbefunde von Patienten mit hirnorganischem Anfallsleiden wurden - soweit sie nicht aufgrund der Kriterien 2 und 3 ausgeschlossen waren - nur dann in das Normalkollektiv eingeschlossen, falls keine eindeutige Ursache für das Anfallsleiden zu erheben war und der letzte Anfall in einem ausreichenden zeitlichen Abstand (9) zur Lumbalpunktion aufgetreten war.

Bei Anwendung all dieser Auswahlkriterien konnten dann die Liquorelektrophoresen von 140 Patienten zur Normalwertbestimmung herangezogen werden. Wurde ein Patient wiederholt punktiert, so wurde nur eine Liquoruntersuchung zur Normalwertermittlung benutzt. Es handelte sich bei den 140 Patienten sowohl um solche mit neurologischen (chronischer Kopfschmerz, 
unklarer Schwindel, familiärer Tremor, periphere traumatische Nervenschädigung u. a.) als auch mit psychiatrischen (Schizophrcnie, neurotische Depression, endogene Depression, Neurose u. a.) oder mehr internistischen Erkrankungen (synkopale Anfälle u. a.).

\section{Ergebnisse und Diskussion}

\section{Reproduzierbarkeit der angewandten Metboden}

Die Reproduzierbarkeit der für die Ermittlung der Normalwerte angewandten Methoden wurde bestimmt. Dazu wurde die Gesamteiweißbestimmung an 47 verschiedenen Tagen und die elektrophoretische Auftrennung an 16 verschiedenen Tagen bei der gleichen Liquorkontrollprobe ${ }^{8}$ ) in Doppelbestimmungen durchgeführt. Aus der Differenz der Doppelbestimmungen wurde die Streuung in der Serie und aus dem jeweils ersten Wert der Doppelbestimmungen die Streuung von Tag zu Tag berechnet.

$\mathrm{Da}$ keine scharfe Trennung $\operatorname{der} \beta$ - und $\tau$-Fraktionen bei der Elektrophorese der Liquorkontrollprobe erfolgte, werden sie in Tabelle 1 als eine $\beta$-Fraktion angegeben.

Tab. 1

Reproduzierbarkeit der Liquorgesamteiweißbestimmung ( $n=47$ Tage) und der elektrophoretischen Fraktionen ( $n=16$ Tage)

\begin{tabular}{|c|c|c|c|}
\hline Bestandteil & $\begin{array}{l}\text { Statistische } \\
\text { Kenngröße }\end{array}$ & $\begin{array}{c}\text { Streuung } \\
\text { von Tag zu Tag }\end{array}$ & $\begin{array}{c}\text { Streuung } \\
\text { in der Serie }\end{array}$ \\
\hline Gesamteiweiß & $\begin{array}{l}\bar{x}(\mathrm{mg} / \mathrm{l}) \\
\mathrm{s}(\mathrm{mg} / \mathrm{l}) \\
\mathrm{v}(\%)\end{array}$ & $\begin{array}{r}951,6 \\
64,1 \\
6,7\end{array}$ & $\begin{array}{r}951,6 \\
26,6 \\
2,8\end{array}$ \\
\hline Präalbumin & $\begin{array}{l}\bar{x}(\%) \\
\mathbf{s}(\%) \\
\text { v }(\%)\end{array}$ & $\begin{array}{c}2,13 \\
0,50 \\
23,5\end{array}$ & $\begin{array}{l}2,13 \\
0,14 \\
6,6\end{array}$ \\
\hline Albumin & $\begin{array}{l}\bar{x}(\%) \\
\text { s }(\%) \\
\text { v }(\%)\end{array}$ & $\begin{array}{c}61,84 \\
3,01 \\
4,9\end{array}$ & $\begin{array}{c}61,84 \\
1,59 \\
2,6\end{array}$ \\
\hline$\alpha_{1}$-Globulin & $\begin{array}{l}\bar{x}(\%) \\
\text { s (\%) } \\
\text { v (\%) }\end{array}$ & $\begin{array}{c}4,81 \\
0,85 \\
17,7\end{array}$ & $\begin{array}{l}4,81 \\
0,44 \\
9,1\end{array}$ \\
\hline$\alpha_{2}$-Globulin & $\begin{array}{l}\bar{x}(\%) \\
\text { s }(\%) \\
\text { v }(\%)\end{array}$ & $\begin{array}{c}5,78 \\
0,66 \\
11,4\end{array}$ & $\begin{array}{l}5,78 \\
0,53 \\
9,2\end{array}$ \\
\hline$\beta$-Globulin & $\begin{array}{c}\bar{x}(\%) \\
\text { s }(\%) \\
\text { v }(\%)\end{array}$ & $\begin{array}{c}11,50 \\
1,83 \\
15,9\end{array}$ & $\begin{array}{c}11,50 \\
1,00 \\
8,7\end{array}$ \\
\hline$\gamma$-Globulin & $\begin{array}{c}\overline{\mathbf{x}}(\%) \\
\text { s }(\%) \\
\text { v }(\%)\end{array}$ & $\begin{array}{c}13,91 \\
1,86 \\
13,4\end{array}$ & $\begin{array}{c}13,91 \\
1,13 \\
8,1\end{array}$ \\
\hline
\end{tabular}

8) Spinal Fluid Control, Hyland Laboratories, Los Angeles (USA)

\section{Normalwertc}

Das Ergebnis der Untersuchung von 140 Probanden (60 Frauen, 80 Männer) ist in Tabelle 2 zusammengefaßt.

Zur Beschreibung eines Normalkollektivs werden häufig arithmetisches Mittel ( $(\bar{x})$ und Standardabweichung (s) benutzt, wobei übereinkunftgemäß der Normalbereich durch $\overline{\mathrm{x}} \pm 2$ s festgelegt wird. Innerhalb der so gewonnenen unteren und oberen Grenze des Normalkollektivs liegen allerdings nur dann annähernd $96 \%$ aller Werte, wenn dic Annahme der Normalverteilung nicht im Widerspruch zu der vorliegenden empirischen Verteilung steht. Ein anderes, rein deskriptives Vorgehen ist die Beschreibung eines Kollektivs durch Median und Perzentilgrenzen. In Tabelle 2 sind beide Verfahren zur Charakterisierung des Normalbereichs benutzt. Bei der von uns untersuchten Stichprobe stimmen Median und arithmetisches Mittel weitgehend überein. Das gleiche gilt für die Grenzen des Normalbereichs, die durch $\overline{\mathrm{x}} \pm 2 \mathrm{~s}$ oder Perzentile festgelegt wurden. Daraus folgt, daß die empirische Verteilung der analysierten Bestandteile als weitgehend symmetrisch angesehen werden kann. Auffallend ist, daß die methodische Streuung (Tab. 1) in derselben Größenordnung liegt wie die Gesamtstreuung des Normalkollektivs (Tab. 2). Auf einen Vergleich der ermittelten Normalwerte mit den in der Literatur mitgeteilten wird verzichtet, da eine Úbereinstimmung in der angewandten Methodik (Meßverfahren, Zusammensetzung des Standards) und in den Auswahlkriterien für das Ziehen der Stichprobe in keinem Fall gegeben ist. Im einzelnen ist die Methodenabhängigkeit der Normalwerte für das Liquorgesamteiweiß und die elektrophoretischen Fraktionen an anderer Stelle diskutiert (10-12).

In einer methodischen Untersuchung über verschiedene Verfahren zur Konzentrierung des Liquors konnten KapLan und Johnstone (13) sowie Windisch und BRACKEN (14) zeigen, daß der Hauptanteil der methodischen Streuung bei der Elektrophorese nicht durch das von ihnen benutzte Einengungsverfahren bedingt ist.

Tab. 2

Normalbereiche für das Liquorgesamteiweiß und die elektrophoretischen Fraktionen

\begin{tabular}{|c|c|c|c|c|c|c|c|c|c|c|}
\hline $\begin{array}{l}\text { Stich- } \\
\text { probe }\end{array}$ & $\begin{array}{l}\text { Statist. } \\
\text { Kenngröße }\end{array}$ & $\begin{array}{l}\text { Gesamt- } \\
\text { eiweiß } \\
\text { (mg/l) }\end{array}$ & $\begin{array}{l}\text { Präalbumin } \\
(\%)\end{array}$ & $\underset{(\%)}{\text { Albumin }}$ & $\underset{(\%)}{\alpha_{1} \text {-Globulin }}$ & $\underset{(\%)}{\alpha_{2}-\text { Globulin }}$ & $\underset{(\%)}{\beta-G \text { lobulin }}$ & $\underset{(\%)}{\tau-G l o b u l i n}$ & $\begin{array}{c}\beta+\tau \text {-Glo- } \\
\text { bulin (\%) }\end{array}$ & $\begin{array}{c}\boldsymbol{\gamma} \text {-Globulin } \\
(\%)\end{array}$ \\
\hline $\begin{array}{l}\text { Männer } \\
\mathrm{n}=80\end{array}$ & $\begin{array}{c}\overline{\mathbf{x}} \\
\overline{\mathbf{x}} \pm 2 s \\
\mathbf{s} \\
\mathbf{v}(\%) \\
\text { Median } \\
\text { 96-Perzen- } \\
\text { tilgrenzen }\end{array}$ & $\begin{array}{c}370 \\
188-522 \\
91,0 \\
24,6 \\
366 \\
550 \\
185\end{array}$ & $\begin{array}{c}2,8 \frac{4,9}{1,10} \\
21,9 \\
5 \\
7 \\
3\end{array}$ & $\begin{array}{c}63,7 \\
55,2 \frac{72,2}{4,25} \\
6,7 \\
64 \\
71 \\
56\end{array}$ & $\begin{array}{c}3,1 \frac{3,4}{1,7} \\
1,15 \\
34,4 \\
3 \\
6 \\
2\end{array}$ & $\begin{array}{c}2,9-9,6,9 \\
1,00 \\
20,8 \\
5 \\
7 \\
3\end{array}$ & $\begin{array}{c}8,8 \\
6,1 \frac{11,4}{1,30} \\
15,0 \\
9 \\
11 \\
6\end{array}$ & $\begin{array}{c}6,1 \\
2,9-9,4 \\
1,65 \\
26,7 \\
6 \\
9 \\
3\end{array}$ & $\begin{array}{c}14,9 \\
10,0-19,7 \\
2,40 \\
16,3 \\
15 \\
21 \\
11\end{array}$ & $\begin{array}{c}8,5 \frac{8}{11}, 3 \\
1,50 \\
22,8 \\
8 \\
12 \\
5\end{array}$ \\
\hline $\begin{array}{l}\text { Gesamt- } \\
\text { kollektiv } \\
n=140\end{array}$ & $\begin{array}{c}\bar{x} \\
\bar{x} \pm 2 s \\
s \\
v(\%) \\
\text { Median } \\
\text { 96-Perzen- } \\
\text { tilgrenzen }\end{array}$ & $\begin{array}{c}356 \\
174 \frac{538}{91} \\
25,6 \\
360 \\
520 \\
173\end{array}$ & $\begin{array}{c}5,2 \\
2,5-7,9 \\
1,35 \\
25,7 \\
5 \\
8 \\
3\end{array}$ & $\begin{array}{c}62,7 \\
53,0-72,4 \\
4,85 \\
7,7 \\
63 \\
71 \\
54\end{array}$ & $\begin{array}{c}3,6 \\
1,1 \frac{6,1}{1,25} \\
34,8 \\
3 \\
6 \\
2\end{array}$ & $\begin{array}{c}5,0 \\
2,7-7,3 \\
1,15 \\
22,9 \\
5 \\
8 \\
3\end{array}$ & $\begin{array}{c}8,2 \frac{8,8}{11}, 5 \\
1,35 \\
15,0 \\
9 \\
11 \\
6\end{array}$ & $\begin{array}{c}6,1 \\
2,8-9,3 \\
1,60 \\
26,7 \\
6 \\
9 \\
3\end{array}$ & $\begin{array}{c}14,9 \\
10,0-19,8 \\
2,45 \\
16,4 \\
15 \\
20 \\
10\end{array}$ & $\begin{array}{c}8,6 \\
4,0 \frac{13,2}{2,30} \\
26,6 \\
8 \\
14 \\
5\end{array}$ \\
\hline
\end{tabular}




\section{Altersabbängigkeit}

Es wurden die Produkt-Moment-Korrelationskoeffizienten für das Gesamteiweiß, die Gesamt- $\beta$-Fraktion und die $\gamma$-Fraktion in Abhängigkeit vom Alter ermittelt, und zwar für Männer und Frauen getrennt und für beide Gruppen zusammen (Tab. 3). Das Alter der Probanden liegt zwischen 16 und 74 Jahren.

Tab. 3

Produkt-Moment-Korrelationskoeffizienten des Liquor-Gesamteiweißes, der $\beta$-Fraktion und $\gamma$-Fraktion in Abhängigkeit vom Alter

\begin{tabular}{llcc}
\hline \multicolumn{1}{c}{ Bestandteil } & $\begin{array}{c}\text { Produkt-Moment-Korrelationskoeffizient } \\
\text { Frauen } \\
\mathrm{n}=60\end{array}$ & $\begin{array}{c}\text { Männer } \\
\mathrm{n}=80\end{array}$ & $\begin{array}{c}\text { Gesamt } \\
\mathrm{n}=140\end{array}$ \\
\hline Gesamteiwei & 0,2280 & 0,2523 & 0,2088 \\
$\beta+\tau$-Fraktion & 0,2611 & 0,2266 & 0,2407 \\
$\gamma$-Fraktion & 0,0541 & 0,1930 & 0,1334 \\
\hline
\end{tabular}

Mit Ausnahme der $\gamma$-Fraktionen sind die berechneten Korrelationskoeffizienten bei der großen Anzahl der Probanden statistisch signifikant von Null verschieden, wenn man Normalverteilung der Werte voraussetzt. Dies deutet auf eine mögliche Abhängigkeit der genannten Größen vom Alter hin (15). Für die Routinediagnostik ist dieser Befund ohne praktische Bedeutung.

\section{Geschlecbtsabbängigleeit}

Bei den in Tabelle 2 angegebenen Normalwerten fällt auf, daß das arithmetische Mittel für das Gesamteiweiß bei den Männern höher liegt als bei den Frauen. Dieser Unterschied ist jedoch statistisch nicht signifikant (Wilcoxon-Test, $\mathrm{p}=0,05)$ (16). Dieses Ergebnis steht in Übereinstimmung mit den Befunden anderer Autoren (17-19).

Die Frage, ob dem gefundenen Unterschied in der oberen Normgrenze bei den untersuchten Stichproben der $\gamma$-Globuline der Männer und Frauen eine allgemeine Bedeutung zukommt, muß offen bleiben. Zwar zeigte die Prüfung auf Verteilungsunterschiede $\left(x^{2}-\right.$ Test) zwischen den $\gamma$-Globulinen der Männer und Frauen eine Signifikanz zum 1\%-Testniveau, die offensichtlich dadurch zustande kommt, daß die Meßskala für die niedrigen Werte bei den Männern stärker besetzt ist als bei den Frauen. Ein Unterschied in der oberen Normgrenze hingegen läßt sich aufgrund des Umfangs der Stichproben statistisch nicht sichern.

Herrn Dr. E. Hansert, Leiter der Biometrischen Abteilung des Max-Planck-Instituts für Psychiatrie danken wir für die Hilfe bei der statistischen Auswertung.

\section{Literatur}

1. Orrol-Bosch, A. und K. D. Vorgt, Klin. Wschr. 37, 758 (1959). - 2. KoHN, J., Ärztl. Lab. 10, 233 (1964). - 3. Gilland O., Acta neurol. Scand. 43, 403 (1967). - 4. Marer, K. H. und K. VoGgel, Klin. Wschr. 41, 286 (1963). - 5. ARnold, F., Schweiz. Med. Wschr. 96, 1098 (1966). - 6. Delank, H. W., Dtsch. Zschr. Nervenhk. 174, 429 (1956). - 7. Werse, H. J., Zschr. inn Med. 14, 167 (1959). - 8. WEISE, H. J., Klin. Wschr. 38, 1040 (1960). - 9. SCHEID, W., Die cerebralen Anfallsleiden, Lehrbuch der Neurologie, 3. Auflage, Thieme-Verlag, Stuttgart (1968). 10. Bauer, H und I. ANgelstein, Klin. Wschr. 30, 277 (1952). 11. Bauer, H., Internist 2, 85 (1961). - 12. Delank, H. W., Das EiweiBbild des Liquor cerebrospinalis und seine klinische $\mathrm{Be}$ deutung, Dr. Dietrich Steinkopff Verlag, Darmstadt (1965). 13. Kaplan, A. und M. Johnstone, Clin. Chem. (New York) 12, 712 (1966). - 12. WrNDISCH, R. M. und M. M. BRACKEN, Clin. Chem. (New York) 16, 416 (1970). - 15. Pryce, J. D., P. W. GANT und K. J. Saur, Clin. Chem. (New York) 16, 562 (1970). 16. BenNetT, C. A. und N. L. FrankLIN, Statistical Analysis in Chemistry and the Chemical Industry, 3. Auflage, John Wiley \& Sons, Inc. New York, London (1963). - 17. RrEDER, H. P. und R. Wüthrich, Klin. Wschr. 40, 1070 (1962). - 18. RIEDER, H. P., Klin. Wschr. 44, 1036 (1966). - 19. Schuller, E., C. RonQues und M. LoridaN, Wien. Zschr. Nervenhk./Suppl. II, 104 (1969).

\section{Dr. Peter Doert}

Klin. Chem. Abt. d. MPI für Psychiatrie 8 München 23

Kraepelinstr. 10 


\section{Laborautomatisation - konsequent unind zukunftssicher}

\section{Direkte Probenidentifikation} On-line-Meßwerterfassung Real-time-Datenverarbeitung
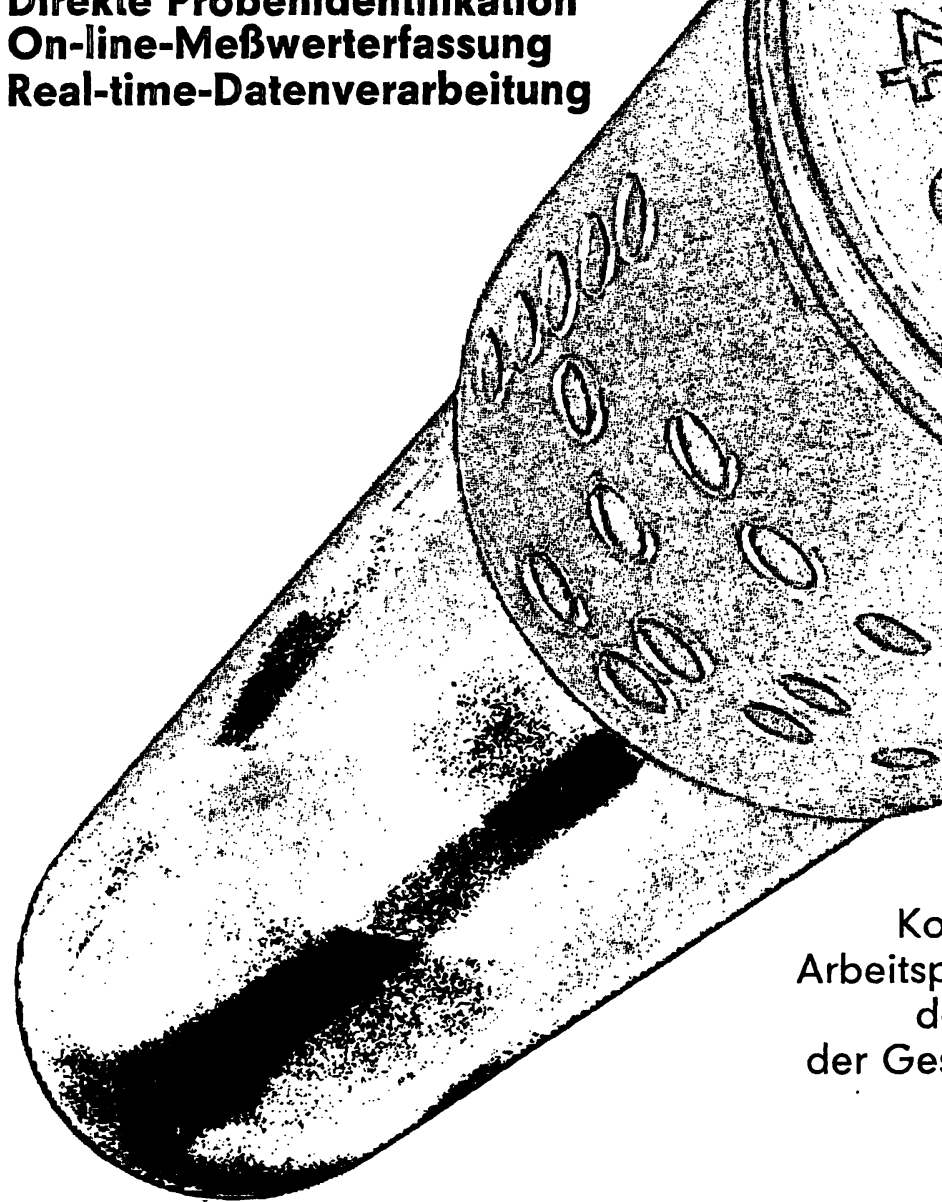

(5)
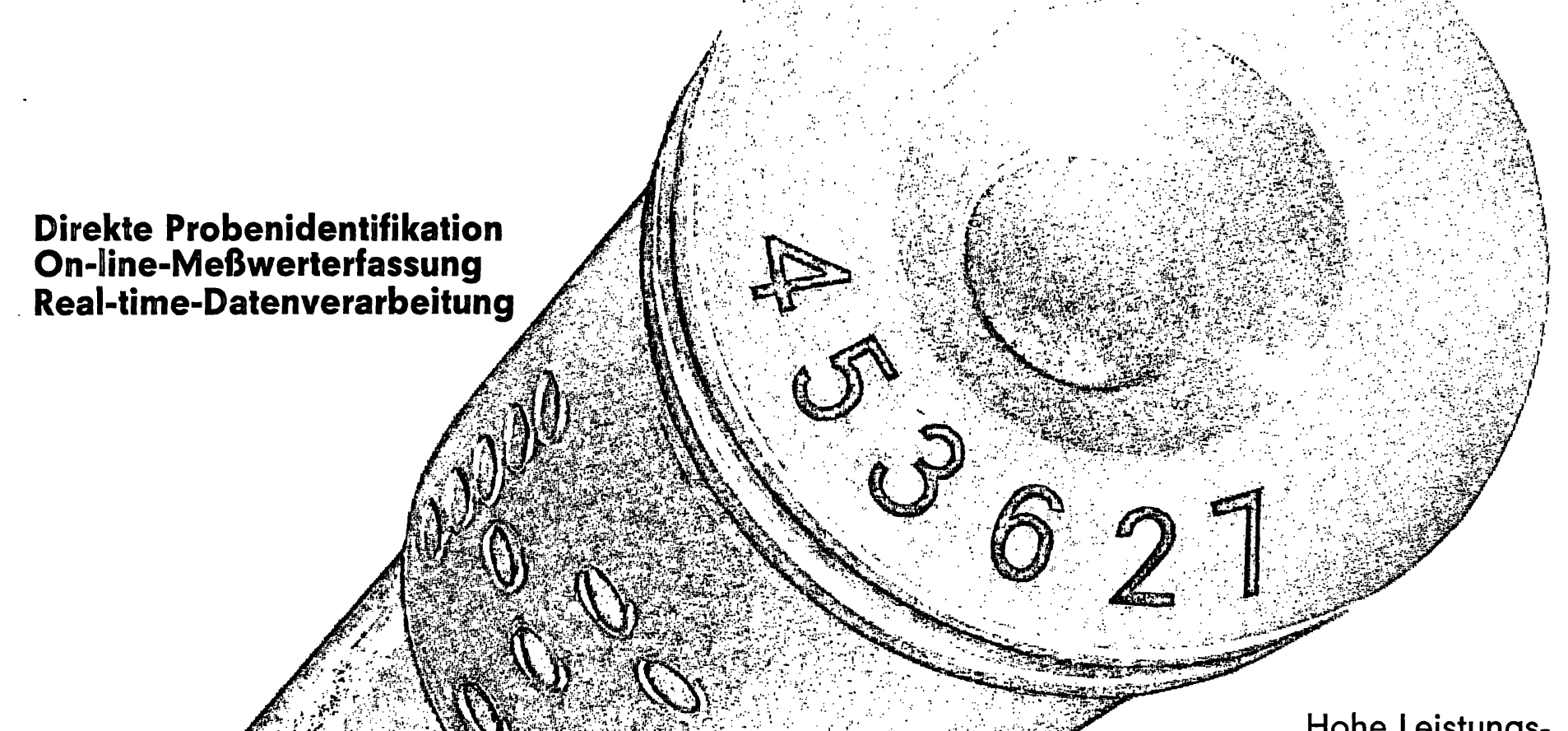

S

Hohe Leistungsfähigkeit des Labors durch effektiveren Einsatz seiner

Fachkräfte und wirtschaftlich optimale Ausnutzung der Analysen- und Meßgeräte. Termingerechte Erledigung aller

Anforderungen mit vielseitiger automatischer Kontrolle und sicherer Identifikation der Meßdaten. Arbeitsplatz- und patientenorientierte Zusammenstellung der automatisch verarbeiteten Daten und Ausgabe der Gesamtinformation in übersichtlichen Ergebnislisten.

SIEMENS AKTIENGESELLSCHAFT Bereich Medizinische Technik . Erlangen

\section{mit dem SILAB-System Von Siemens}




\section{eine Tür ging auf zu einem modernen Programm!}

\section{Heißluft-Sterilisatoren Inkubatoren mit Wassermantel wasserfreie Inkubatoren thermostatisierte Wasserbäder}

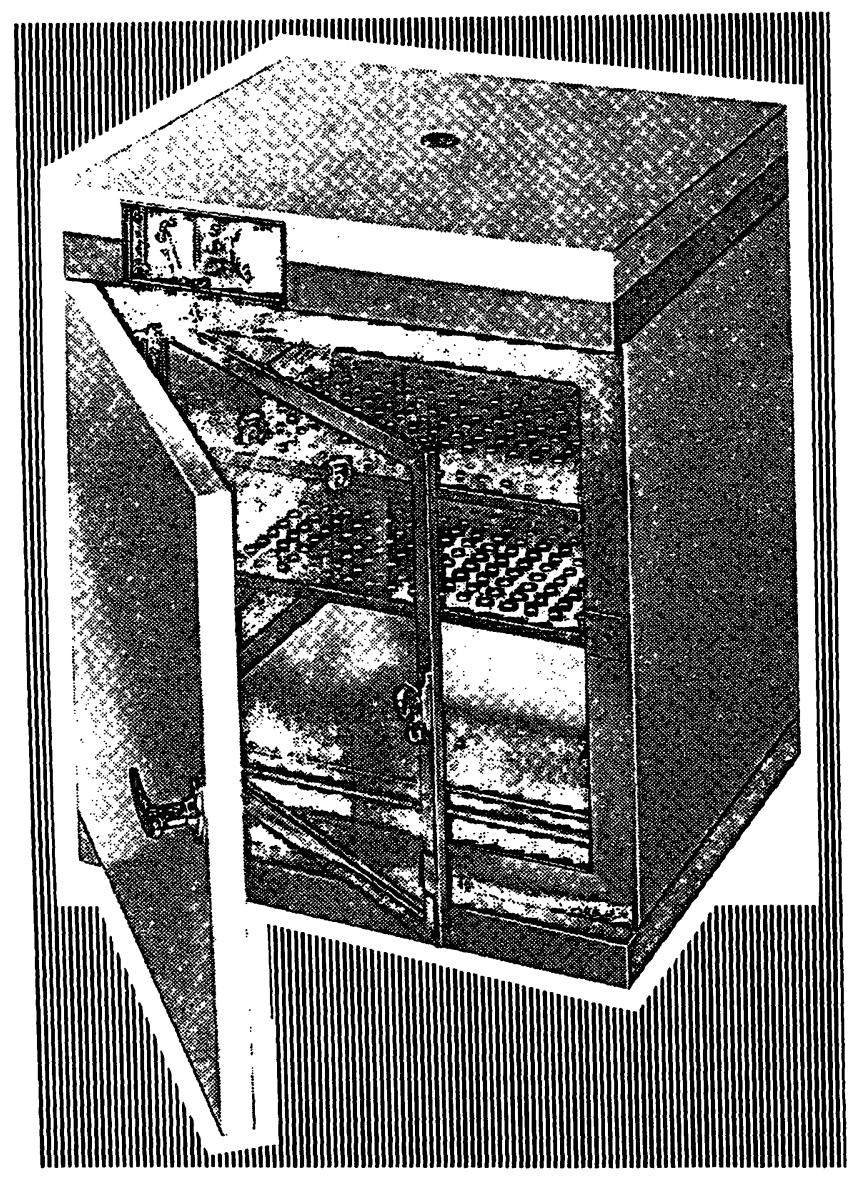

\section{0 \\ korrosionsfreie Laborgeräte}

Unsere Druckschrift STA-71

informiert Sie ausführlich.

WISSENSCHAFTLICHE APPARATE

HORMUTH-VETTER

6908 Wiesloch/Bd., Postfach 1348, Tel. 06222/21 47 6900 Heidelberg 1, Postfach 750, Tel. 0622120045

\section{ARBEITSMETHODEN}

DER MODERNEN NATURWISSENSCHAFTEN

Herausgegeben von Prof. Dr. KuRt FischBrCK

\section{KONRAD DORFNER}

\section{Ionenaustauscher}

\section{Aufláge}

Mit 100 Abbildungen, 27 Tabellen im Text und 1 Tabellenanhang (mit 19 Tabellen). Oktav. XII, 320 Seiten. 1970. Plastikeinband DM 58,-

Nach der erweiterten Einführung wird eine ausführlichere Darstellung det verschiedenen Ionenaustauschertypen sowie ihrer Eigenschaften und Prüfmethoden gegeben. Besonders über die Verwendung der Ionenaustauscher in der Technik wird im einzelnen berichtet, um den Neuentwicklungen gerecht zu werden. So sind die rechnerische Behandlung und die speziellen Verfahren des Festbettverfahrens, die neuesten Entwicklungen der kontinuierlichen Verfahren, die Wasseraufbereitung mit Ionenaustauschern und die Verwendung der Ionenaustauscher zur Abwasserreinigung, Metallgewinnung, Zuckerherstellung und sonstiger technischer Anwendungen in dem vorgegebenen Rahmen so umfassend wie möglich dargestellt worden. Die übrigen Kapitel wurden nach den neuesten Ergebnissen durchgearbeitet, verbessert und ergänzt.

\section{Johannes Fư̈Gge}

\section{Grundlagen der Polarimetrie}

Gerätekunde und Meßtechnik

Oktav. Mit 72 Abbildungen und 28 Tabellen. XII, 159 Seiten. 1970. Plastikeinband DM 48,-

Wurde die Polarimetrie bereits seit langem als analytisches Verfahren, z. B. in Zuckerfabriken und in Betrieben der pharmazeutischen Chemie, angewandt, so hat sie sich in neuerer Zeit auch in der Exforschung von Molekülstrukturen als aufschlußreich erwiesen, besonders seitdem es automatische und Spektralpolarimeter bis ins Ultraviolett gibt. Das. vorliegende Werk informiert über Grundlagen, Meßtechnik und moderne Geräte dieser optischen Methode und berücksichtigt ihren Stand bis in die jüngste Zeit, wobei neben der Analytik auch die Bestimmung der Rotationsdispersion, der magneto-optischen Drehung des Lichts und der Elliptizität, wie sie bei Zirkulardichroismus auftritt, besprochen werden. Photoelektrische Polarimeter und Saccharimeter werden ausführlich behandelt.

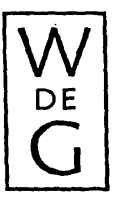

Walter de Gruyter Berlin - New York 


\section{Gastrokamera- Untersuchung}

\section{Grundlagen}

Untersuchungstechnik, Bildbeurteilung, Ergebnisse mit den Referaten des Ersten Gastrokamera-Seminars, 13.-15. Juni 1969, Berlin

\section{Herausgegeben von Dozent Dr. H. Oshima}

Gastprofessor an der Freien Universität Berlin, Nippon-IkaUniversität Tokyo,

Präsident der European Association for Gastrokamera Diagnosis, unter Mitarbeit von Dr. W. Bergrmann, Berlin

Oktav. VIII, 146 Seiten. Mit 33 Abbildungen. 1971.

Kartoniert DM 20,-

Die Gastrokamera-Untersuchung, ein schnelles, einfaches und beschwerdearmes Verfahren zur intragastralen farbigen Fotografie für die Magen-Diagnostik hat in Europa in den letzten Jahren eine zunehmende Bedeutung erlangt.

Vom 13.-15. 6. 1969 wurde das Erste Gastrokamera-Seminar im Klinikum Steglitz der Freien Universität Berlin veranstaltet.

Die verschiedenen Gastrokamera-Modelle, die Untersuchungstechnik und besonders die Beurteilungsmethode sowie -kriterien wurden als Schwerpunkte behandelt.

Durch die Berichte der verschiedenen Untersucher aus 6 europäischen Ländern wurde der derzeitige Stand der GastrokameraDiagnostik in Europa dargelegt.

Die etwa 300 Teilnehmer ạs 9 europäischen Ländern bewiesen das rege Interesse an dieser Untersuchungsart.

An einer weiter zunehmenden Bedeutung dieses diagnostischen Verfahrens ist bei der Häufigkeit von Magenerkrankungen, insbesondere des Magenkrebses nicht zu zweifeln. Dazu soll die Herausgabe dieser Verhandlungsberichte als Leitfaden der Gastrokamera-Untersuchung beitragen.

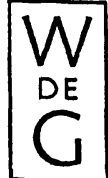

\section{Universelle und spezielle UV-Strahler.* Für jeden Zweck das vorteilhafteste Gerät}

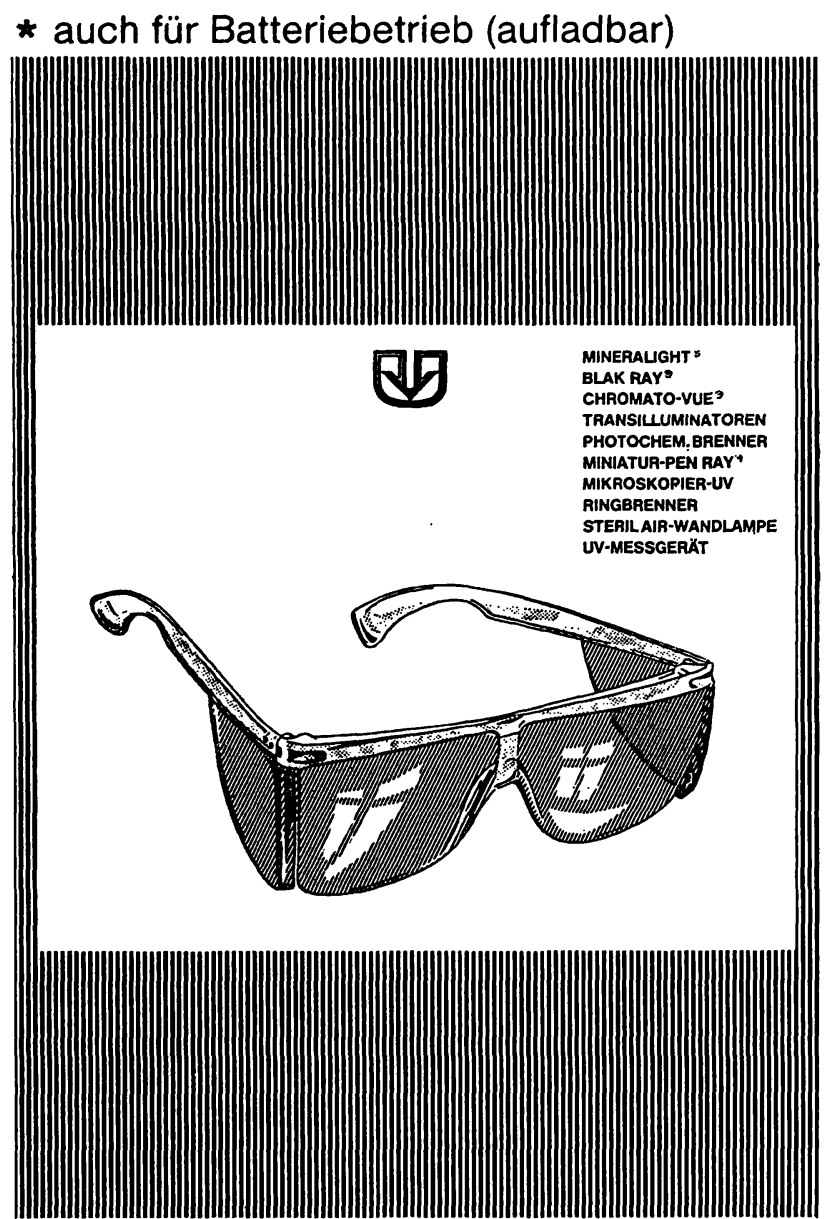

Druckschrift UVC-691: UV f. Chromatographie Druckschrift UV-690: UV f. allgem. Zwecke

Unsere interessanten Druckschriften informieren Sie ausführlich.

WISSENSCHAFTLICHE APPARATE

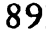

VIDim

6908 Wiesloch/Bd., Postf. 1348, Tel. 06222/2147 6900 Heidelberg 1, Postf. 750, Tel. 06221/20045 


\title{
Proceedings
}

\section{of the International Research Conference on Proteinase Inhibitors}

Munich, November 1970

\author{
Consulting Editors H. Fritz, H. Tschesche
}

XII, 304 pages. 1971. Bound DM 108,-; \$ 31.75

Contents (extract):

Specific Isolation and Modification Methods for Proteinase Inhibitors and Proteinases (Affinity Chromatography);

Chemistry, Biochemistry and Structure of Proteinase Inhibitors from Animal Organs and Secretions, e. g. Human Serum, Colostrum, Bovine Organs, Pancrease, Submandibular Glands, Leeches-Isolation, Ascaris Lumbricoides and Protein. Chemistry and Biology of Proteinase Inhibitors from Soybeans, Lima Beans, Peanuts, Potatoes and Maize;

Studies on the Physiological Functions of these Inhibitors;

Theory of the Enzyme Inhibitor Interaction and Studies on Inhibitory;

Characteristics Structure of Basic Inhibitors of Bovine Organs and some Serine Proteinases (Trypsin, Chymotrypsin, Elastase, Subtilisin e. a.).

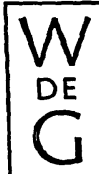

\section{Walter de Gruyter - Berlin - New York}

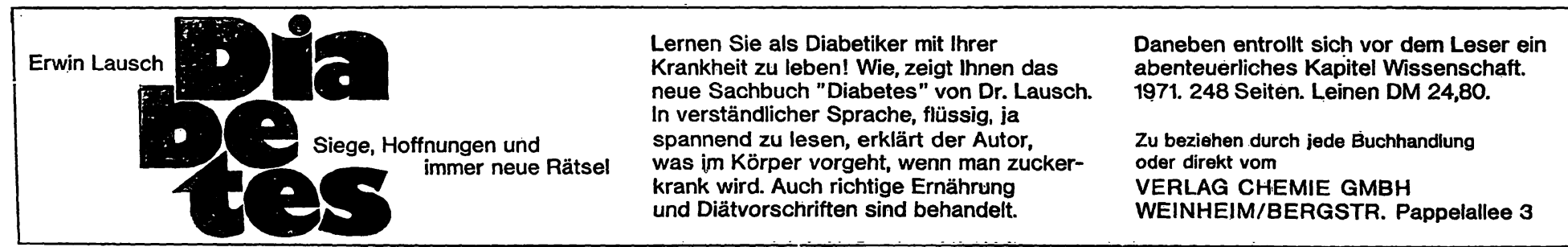

